\title{
INDEX OF SUBJECTS FOR VOLUMES 19 and 20
}

Acne vulgaris, microbiology

VOL PAGE

Adherence

Bord. pertussis to tissue culture cells (assay) 19

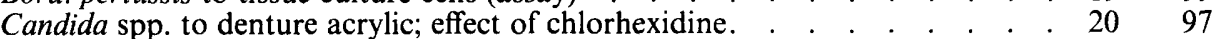

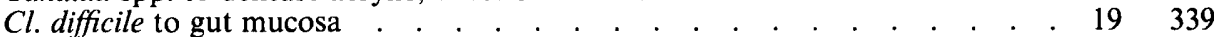

colonisation of intravenous cannulae. . . . . . . . . . . . . . . . . . . . . . . .

Esch. coli to HEp2 cells . . . . . . . . . . . . . . . . . . . . . . . . . . . . 20345

Esch. coli to uro-epithelial cells. . . . . . . . . . . . . . . . . . . . . . . . 2033

Klebsiella, type-3 fimbriae . . . . . . . . . . . . . . . . . . . . . . . . . . . . 20203

S. aureus to silicone polymer . . . . . . . . . . . . . . . . . . . . . . . . . 2020249

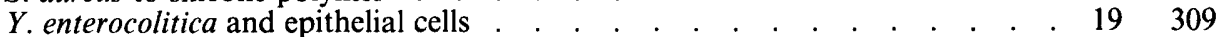

Aeromonas

A. hydrophila, effect of $\mathrm{Fe}^{+++}$and tetracycline . . . . . . . . . . . . 2017

haemagglutinin detection . . . . . . . . . . . . . . . . . . . . . . 19273

Anaerobes (see also Clostridium and Bacteroides)

blood culture media, comparison . . . . . . . . . . . . . . . . . . . . . 19187

media for growth of bacteroides . . . . . . . . . . . . . . . . . . . . . . 19195

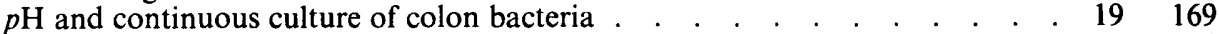

transport media for clinical specimens .

Antibody response to $\mathrm{Q}$ fever . . . . . . . . . . . . . . . . . . . . . . . . . . $\quad . \quad 19281$

Antibiotics and other antibacterial substances

amoxycillin on oral flora. . . . . . . . . . . . . . . . . . . . . . . 19

ampicillin and erythromycin on Legionella. . . . . . . . . . . . . . . . . $\quad$. 19383

chlorhexidine, candida adherence . . . . . . . . . 2097

chlorhexidine, rotavirus morphology. . . . . . . . . . . . . . . . . . . . 20123

ethanol, rotavirus morphology . . . . . . . . . . . . . . . . . . . . . . . . . 2023

glutaraldehyde, rotavirus morphology . . . . . . . . . . . . . . . . . . . . 2023

hypochlorite, rotavirus morphology .

metronidazole in pulmonary trichomoniasis . . . . . . . . . . . . . . . . $20 \quad 1$

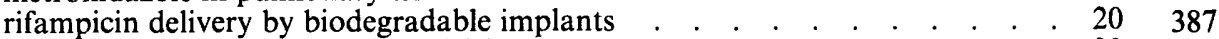

tetracycline, antagonism with $\mathrm{Fe}^{+++}$. . . . . . . . . . . . . . . . . . 2017

tetracycline and experimental candidosis in rats . . . . . . . . . . . . . . . . . 2029

vancomycin in $\mathrm{Cl}$. difficile ileocaecitis in hamsters . . . . . . . . . . . . . . . . 19339

Antibiotic resistance

aminoglycoside, in S. aureus . . . . . . . . . . . . . . . . . . . . . . . . 20169

aminoglycoside, in enterococci . . . . . . . . . . . . . . . . . . . . . . . $\quad$. 20187

-lactamase production in Ps. aeruginosa. . . . . . . . . . . . . . . . . . . . . . . . 1945

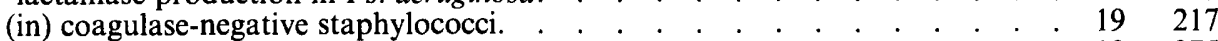

gentamicin and carbenicillin in Ps. aeruginosa. . . . . . . . . . . . . . . . 19375

gentamicin in S aureus . . . . . . . . . . . . . . . . . . . . . . . . . . 2020157

methicillin in S. aureus . . . . . . . . . . . . . . . . . . . . . . . . 2039

methicillin in $S$, aureus . . . . . . . . . . . 20139

methicillin in $S$. aureus $\quad . \quad$.

methicillin in S. aureus and lysogenicity. . . . . . . . . . . . . . . . 20147

penicillin in N. gonorrhoeae (PPNG) . $\quad . \quad$.

penicillin in $S$. aureus . . . . . . . . . . . . . . . . . . . . . . . . . 20325

(in) S. aureus in Australia . . . . . . . . . . . . . . . . . . . . . . . . . . 19137

Antistreptolysin O, false + ve and - ve results . . . . . . . . . . . . . . . . 19

API test for B. anthracis. . . . . . . . . . . . . . . . . . . . . . . . . . . . . . . 20

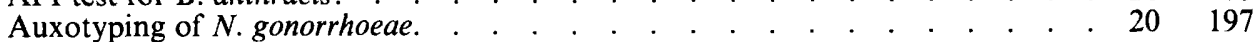

Baboons, intestinal spirochaetosis . . . . . . . . . . . . . . . . . 19359

Bacillus anthracis, identification by API . . . . . . . . . . . . . . . . . . . . . . . . 2075

(also $B$. cereus, B. mycoides, $B$. thuringiensis)

Bacteriophage, lysogenicity in methicillin-resistant $S$. aureus. . . . . . . . 20

Bacteroides

B. fragilis, cryptic plasmids . . . . . . . . . . . . . . . . . . . . . . . . . 2039

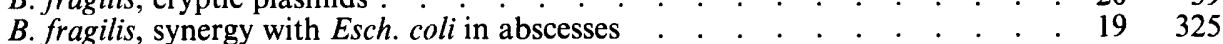

black-pigmented bacteroides, degradation of IgG and complement. . . . . . 1985 
black-pigmented bacteroides, histopathological changes . . . . . . . . . . 20355

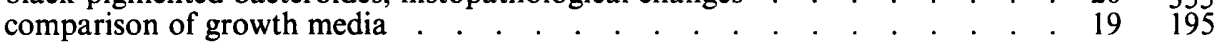

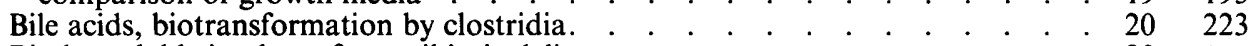

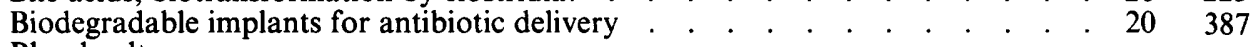

Blood culture

media for oral bacteria

Panmede and Liquoid, effect on Streptobacillus moniliformis isolation. . . . . $\quad 19181$

Bordetella

B. bronchiseptica, virulence for piglets

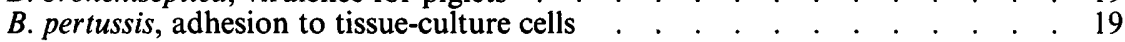

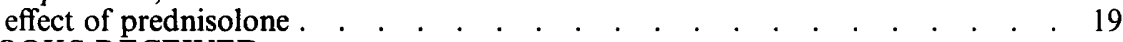

BOOKS RECEIVED

Clinical parasitology (P C Beaver et al.). . . . . . . . . . . . . . . . . . . . . . $\quad$. 19135

Atlas of human parasitology (L R Ash and T C Orihel)

Cell separation-methods and selected applications (edited by $\mathrm{T} \dot{\mathrm{G}}$ Pretlow and $\dot{\mathrm{T}}$ P Pretlow) .

Antibiotic associated diarrhoea and colitis (edited by S P Borriello) $\cdot \cdot \cdot \cdot$

Principles of bacteriology, virology and immunity (Topley and Wilson) (edited by F Brown and Sir G Wilson)

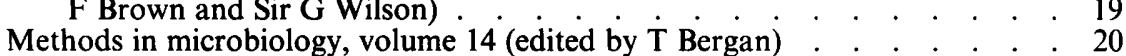

Bacterial vaccines (edited by $\mathbf{R}$ Germanier)

The molecular virology and epidemiology of influenza (edited by $\dot{\mathrm{Sir}} \dot{\mathrm{C}} \dot{\mathrm{H}}$ Stuart-Harris and C W Potter).

Bacterial and viral inhibition and modulation of host defences (edited by $\dot{G}$ Falcone et al.).

Advances in immunology (edited by $\vec{F} \mathbf{J}$ Dixon)

The revival of injured microbes (edited by $\mathrm{M} H \mathrm{H}$ Andrew and $\dot{A} \dot{D}$ Russell)

Introduction to parasitology in tropical Âfrica (F M A Ukoli) . . . . . . 20

Gillies and Dodds bacteriology illustrated (R R Gillies) . . . . . . . . . . . . . . $\quad$. 20

Clinical and oral microbiology (P W Ross and W P Holbrook) . . . . . . . . . . 20

Viral chemotherapy (edited by D Sugar) . . . . . . . . . . . . . . . . 20

Advances in cancer research, volume 41 (edited by $\dot{G}$ Klein and S Weinhouse). $\quad 20$

Models of anaerobic infection (edited by Steering Committee of the Anaerobe Discussion Group: M J Hill et al.)

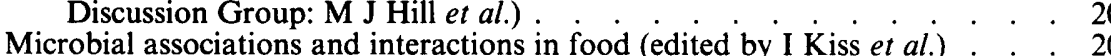

Role of the envelope in the survival of bacteria in infection (edited by $\mathrm{C} \dot{\mathrm{S}} \dot{\mathrm{F}}$ Easmon et al.).

Genetics and biotechnology of bacilli (edited by $\dot{A} \mathbf{T}$ Ganesan and $\mathbf{J} \dot{A}$ Hoch)

Malaria and the red cell (edited by $\mathbf{J} \mathbf{W}$ Eaton and $\mathrm{G} J$ Brewer) ...

Human antiparasitic drugs: pharmacology and usage (edited by $\mathrm{D} \dot{\mathrm{M}} \mathrm{J}$ ames and H M Gilles) . . . . . . . . . . . . . . . . . . . . . . . . 20

\section{Campylobacter}

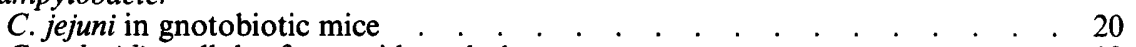

C. pyloridis, cellular fatty acids and ultrastructure

Candida

C. albicans, adherence to acrylic and effect of chlorhexidine . . . . . . . . 20

C. albicans, experimental infection in rat footpad . . . . . . . . . . . . . . . . .220

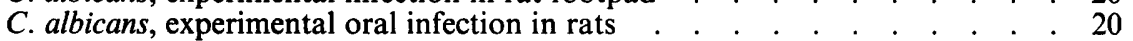

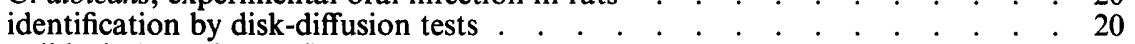

Candidosis (experimental)

in rat footpad . . . . . . . . . . . . . . . . . . . . . . 20

in rat mouth . . . . . . . . . . . . . . . . . . . . . . . . . 20

Capsule expression in S aureus . . . . . . . . . . . . . . . . . . . . . . . . 20

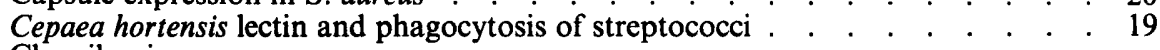

Chemiluminescence

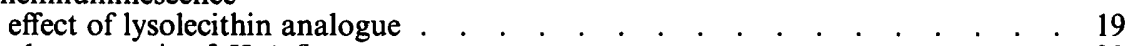

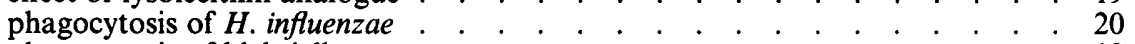

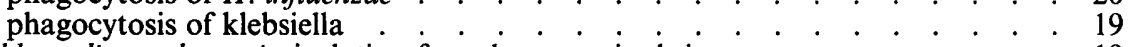

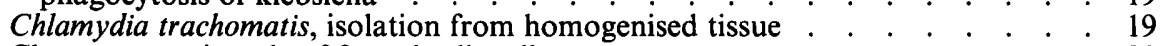

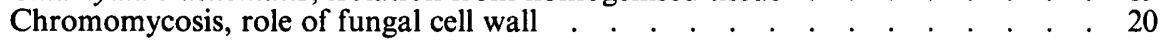


Clostridium

biotransformation of bile acids.

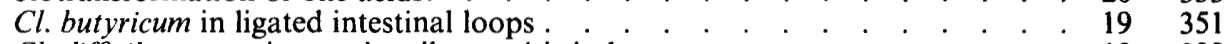

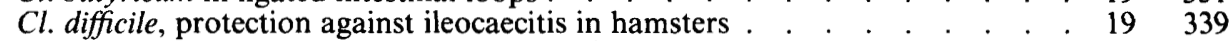

Cl. perenne (and C. jejuni) in gnotobiotic mice . . . . . . . . . . . . . . . . . . 20215

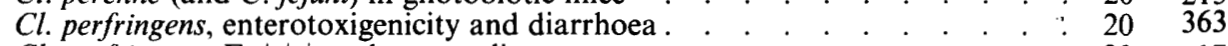

Cl. perfringens, $\mathrm{Fe}^{+++}$and tetracycline . . . . . . . . . . . . . . . . . . 2017

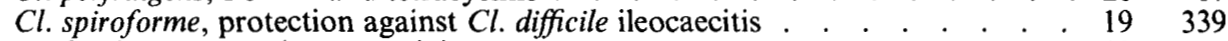

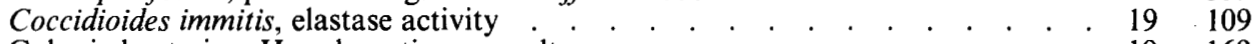

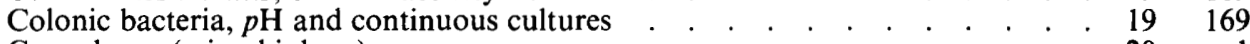

Comedones (microbiology). . . . . . . . . . . . . . . . . . . . . . . . . . . . 201

Complement

degradation by black-pigmented bacteroides . . . . . . . . . . . . . . 1985

CFT in Q fever . .

Conjugates and aminoglycoside-resistance transfer in $S$. aureus. . . . . . . . . 20169

Continuous culture of colon bacteria, $p \mathrm{H}$ effect . . . . . . . . . . . . . . . . . . . $\quad$. $\quad . \quad 19169$

Cooked-meat broth for transport of specimens . . . . . . . . . . . . . . . . . . . . $\quad . \quad 20373$

Coxiella burneti, antibody response . . . . . . . . . . . . . . . . . . . . . . . . . . 19281

Echinococcus, indirect haemagglutination test . . . . . . . . . . . . . . . . . . . . $\quad 1995$

Elastase of Coccidioides immitis . . . . . . . . . . . . . . . . . . . . . . . . . . . . . . . . . 1910

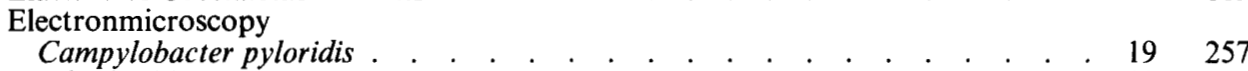

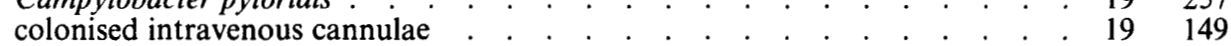

fimbriae of enterobacteria

fimbriae of Enterobacter spp. . . . . . . . . . . . . . . . . . . . . . . . . 1935

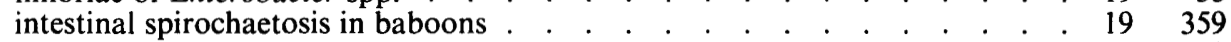

Legionella spp. . . . . . . . . . . . . . . . . . . . . . . . . . . . . . . . . . 1938

Ps. aeruginosa surface. . . . . . . . . . . . . . . . . . . . . . . . . . . . 19375

Pasteurella haemolytica surface . . . . . . . . . . . . . . . . . . . . . . . . 1925

rotavirus . . . . . . . . . . . . . . . . . . . . . . . . 20123

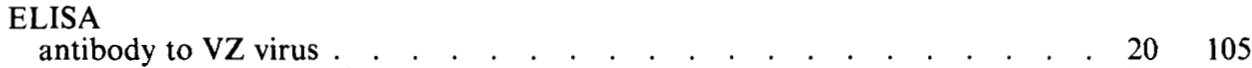

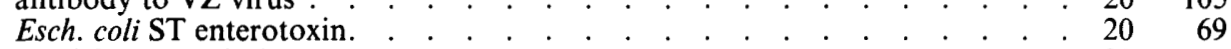

Enteral feeding solutions . . . . . . . . . . . . . . . . . . . . . . . . . . . . 2063

Enterobacter cloacae, in enteral feeds

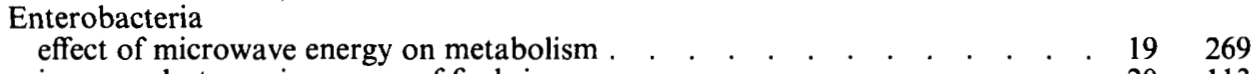

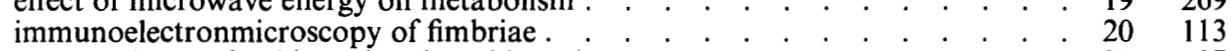

Enterococci, transferable aminoglycoside resistance $\quad . \quad$. . . . . . . . . . . . . . 20187

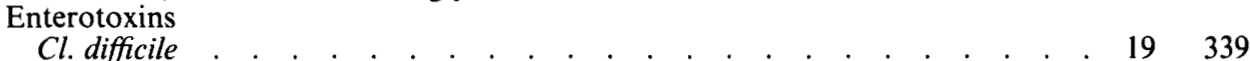

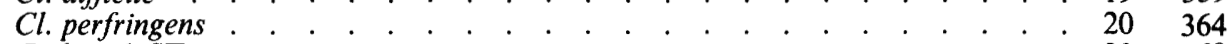

Esch. coli ST. . . . . . . . . . . . . . . . . . . . . . . . . . . . . . . 206

Y.enterocolitica. . . . . . . . . . . . . . . . . . . . . . . . . . . . 19297

Erysipelothrix spp., $\mathrm{Fe}^{++}$and tetracycline . . . . . . . . . . . . . . . . . . . . . $20 \begin{array}{rr}2 & 17\end{array}$

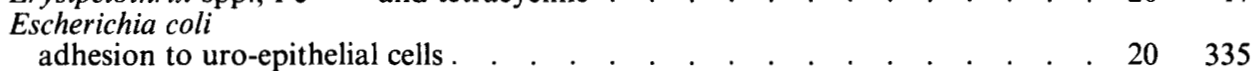

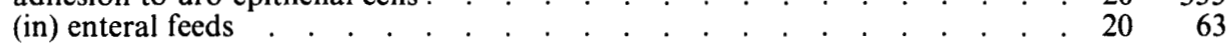

phagocytosis. . . . . . . . . . . . . . . . . . . . . . . . . . . 19115

ST enterotoxin assay .

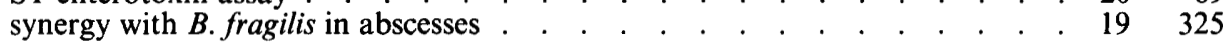

traT gene and serum resistance. . . . . . . . . . . . . . . . . . . . . . . . . . 196

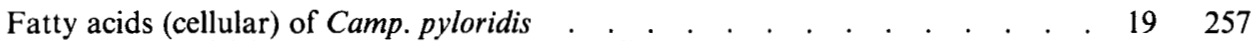

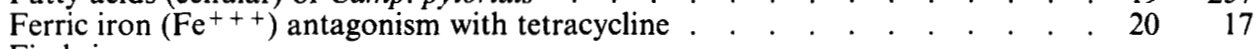

Fimbriae

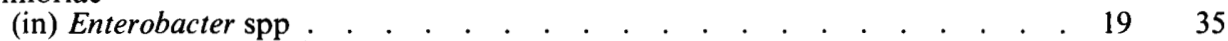

type 3 in enterobacteria . . . . . . . . . . . . . . . . . . . . . . . . . . . . . . 20113

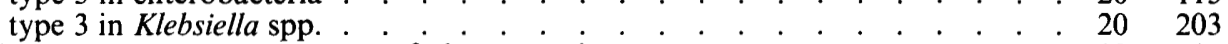

Flow cytometry, measurement of phagocytosis . . . . . . . . . . . . . . . . . 1911 
Fluorescent antibody tests

FAMA for antibodies to VZ virus . . . . . . . . . . . . . . . . . . . . 20105

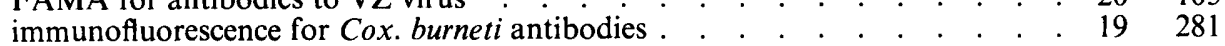

Fungal cell wall in chromomycosis . . . . . . . . . . . . . . . . . . . . 2029

Gastric mucosa-associated campylobacter . . . . . . . . . . . . . . . . . . 19257

Gnotobiotic animals

campylobacters in mice . . . . . . . . . . . . . . . . . . . . 20215

yersiniae in piglets . . . . . . . . . . . . . . . . . . . . . . . . . . . . . . . 1929

Gonococcus (see N. gonorrhoeae)

Haemagglutination (indirect) test for echinococcus . . . . . . . . . . . 1995

Haemagglutinins

Aeromonas . . . . . . . . . . . . . . . . . . . . . . . 19273

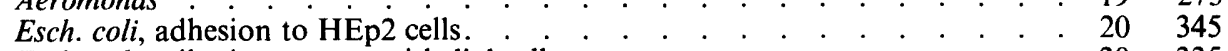

Esch. coli, adhesion to uro-epithelial cells . . . . . . . . . . . . . . . . . . . . . 20335

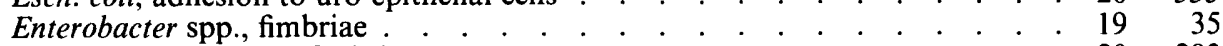

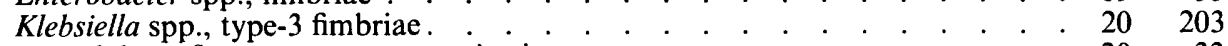

Haemophilus influenzae, serum opsonisation . . . . . . . . . . . . . . . . . . . . . 2033

Heavy metals, resistance in S. aureus . . . . . . . . . . . . . . . . . . . . . . . . . . 19137

Heavy metals, resistance in S. aureus

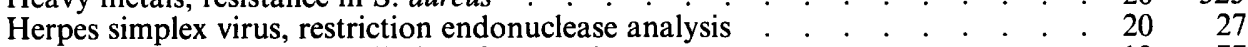

Hydrogen peroxide, susceptibility of Myco. leprae . . . . . . . . . . . . . . . . . . . . .. .1977

Hydrophobicity of $Y$. enterocolitica . . . . . . . . . . . . . . . . . . . . . . . . 19309

Immune response to Noc. asteroides in mice . . . . . . . . . . . . . . . . . . . 20255

Immunoassays of Myco. bovis . . . . . . . . . . . . . . . . . . . . . . . . . 19367

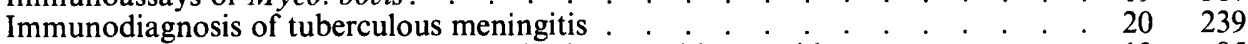

Immunoglobulins, degradation by black-pigmented bacteroides . . . . . . . . . . 1985

Implants, adhesion of S. aureus . . . . . . . . . . . . . . . . . . . . . . . . . . . $\quad .2020$

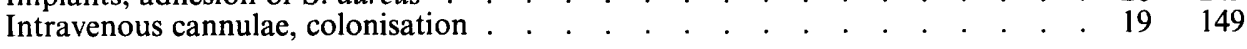

Jet injection, risks of virus transmission . . . . . . . . . . . . . . . . . . . 20393

Klebsiella

$K$. aerogenes in enteral feeds . . . . . . . . . . . . . . . . . . . 2063

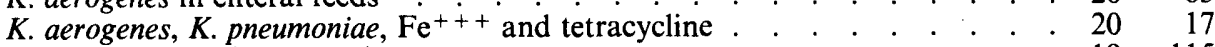

K. pneumoniae, phagocytosis . . . . . . . . . . . . . . . . . . . . 19115

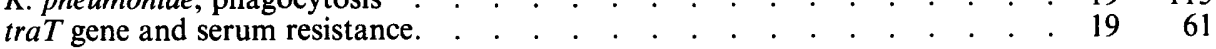

Lectin from Cepaea hortensis and phagocytosis . . . . . . . . . . . . . . . . . . 19317

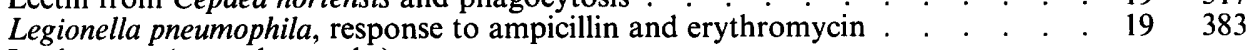

Leukocytes (see polymorphs)

Lipopolysaccharides and colony variants of $N$. gonorrhoeae . . . . . . . . . . . . 19203

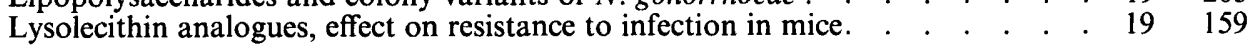

Macrophages, susceptibility of Myco. leprae . . . . . . . . . . . . . . . . . . . . . 1977

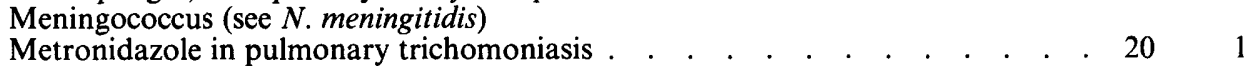

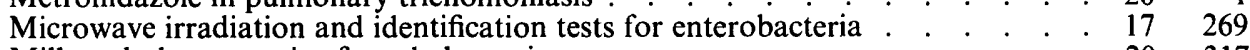

Milk and phagocytosis of staphylococci . . . . . . . . . . . . . . . . . . 20317

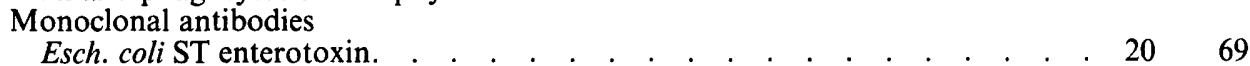

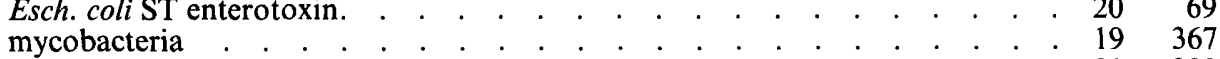

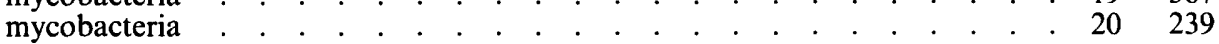

Mycobacteria

antigen and antibody detection in CSF . . . . . . . . . . . . . . . . . . . . 20239

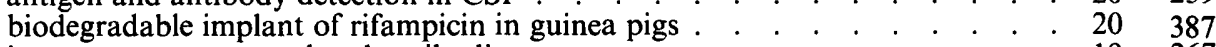

immunoassays, monoclonal antibodies . . . . . . . . . . . . . . . . . . . . . 19267

inhibition by viridans streptococci

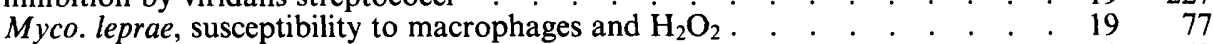

shared antigen and cell-mediated immunity . . . . . . . . . . . . . . . . 1969 
Necrotising enterocolitis, $C l$. butyricum . . . . . . . . . . . . . . . . . . . . . . . $\quad$. 19351

Neisseriae

N. gonorrhoeae, auxotyping and penicillin resistance (PPNG) . . . . . . . 20197

$N$. gonorrhoeae, cell-envelope phenotype, site of infection and serogroup. . . . $20 \quad 379$

N. meningitidis, colony variants (OMP, LPS) . . . . . . . . . . . . . . . . . $\quad$. 19203

N. meningitidis, phagocytosis . . . . . . . . . . . . . . . . . . . . . . . . . . . . . .919

Nocardia asteroides, immune response in mice . . . . . . . . . . . . . . . . . 20255

Nuclease neutralisation test for staphylococci $\quad . \quad$. . . . . . . . . . . . . . . 20392

Nucleic acid-binding compound, resistance in staphylococci. . . . . . . . . . . . $\quad$. 20139

OBITUARY NOTICE, J H Bowie . . . . . . . . . . . . . . . . . . . . . . . $\quad$. 19131

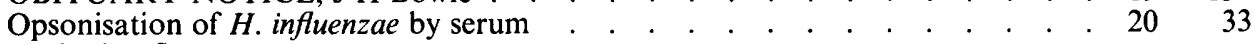

Oral microflora

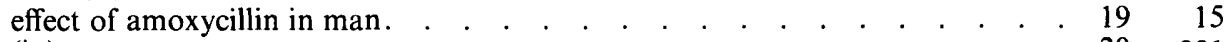

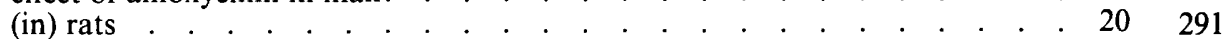

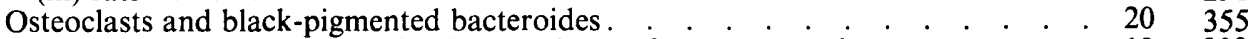

Outer membrane proteins and colony variants of N. meningitidis . . . . . . . 19203

Panmede (in blood cultures)

isolation of Streptobacillus moniliformis . . . . . . . . . . . . . . . . . . . . . . . . 19181

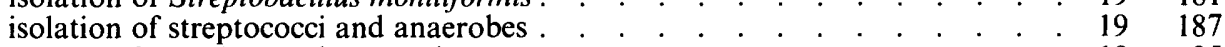

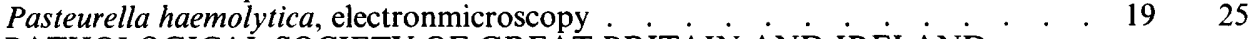

PATHOLOGICAL SOCIETY OF GREAT BRITAIN AND IRELAND

Proceedings of 150 th meeting .

Proceedings of 151 st meeting . . . . . . . . . . . . . . . . . . . . . $19^{\mathrm{i}}$

Phagocytosis

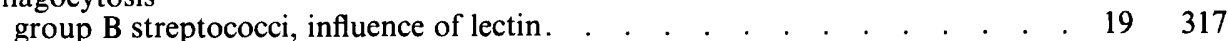

H. influenzae. . . . . . . . . . . . . . . . . . . . . . . . . . . . . 2033

Klebsiella spp. . . . . . . . . . . . . . . . . . . . . . . . . . . . . . . . . 19333

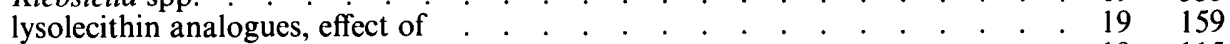

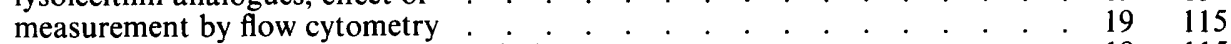

phagosomal $\mathrm{pH}$ and bacterial degradation . . . . . . . . . . . . . . . 19115

staphylococci . . . . . . . . . . . . . . . . . . . . . . . . . . . 20317

Pityrosporum spp. in acne vulgaris . . . . . . . . . . . . . . . . . . . . . . . . $20 \quad 1$

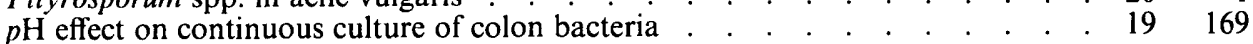

Plasmids $\quad \begin{array}{ll}20 & 39\end{array}$

$\begin{array}{lllllllllllllllllll}\text { B. fragilis, cryptic } & . \\ \text { enterobacteria, trat gene and serum resistance } & . & . & . & . & . & . & . & . & . & . & . & . & . & 20 & 39 \\ \end{array}$

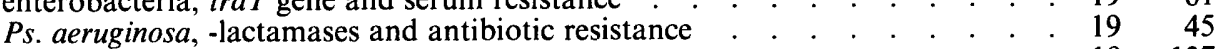

S. aureus, antibiotic and heavy metal resistance . . . . . . . . . . . . . ..$~ 19137$

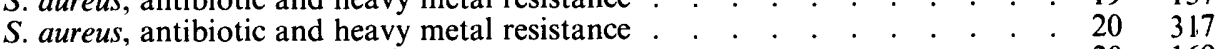

$S$. aureus, aminoglycoside resistance . . . . . . . . . . . . . . . . . . . . . . . 2020

S. aureus, methicillin resistance .

$S$. aureus, methicillin and gentamicin resistance . . . . . . . . . . . . . . . . . 20157

S. aureus, methicillin and nucleic acid-binding compounds resistance . . . . . 20139

Yersinia spp., protection and virulence . . . . . . . . . . . . . . . . . . . . . . 19237

Y. enterocolitica. . . . . . . . . . . . . . . . . . . . . . . . . . . 19297

Polymorphs (and)

black-pigmented bacteroides

$\begin{array}{lllllllllllllllllllllllll}\text { Klebsiella } \operatorname{spp} & . & . & . & . & . & . & . & . & . & . & . & . & . & . & . & . & . & . & . & . & . & . & 19 & 333 \\ \text { lysolecithin analogues. } & . & . & . & . & . & . & . & . & . & . & . & . & . & . & . & . & . & . & . & 19 & 159\end{array}$

phagocytosis, $p \mathrm{H}$ and bacterial degradation $\quad . \quad$. $\quad . \quad$. . . . . . . . . . . . . $\quad$. 19115

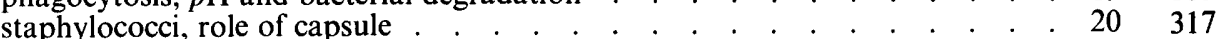

Prednisolone, effect on Bord. pertussis toxicity . . . . . . . . . . . . . . . . . . . . . . . 19391

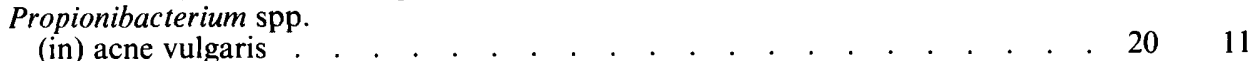

$\begin{array}{llllllllllllllllllllll}\text { (in) acne vulgaris } & . & . & . & . & . & . & . & . & . & . & . & . & . & . & . & . & . & . & . & 20 & 11 \\ \text { PAGE of whole-cell proteins } & . & . & . & . & . & . & . & . & . & . & . & . & . & . & . & . & . & 19 & 9\end{array}$

Proteus spp. 
$\mathrm{Fe}^{++}$and tetracycline . . . . . . . . . . . . . . . . . . . . . . . . 20 . 2011

traT gene and serum resistance. . . . . . . . . . . . . . . . . . . . . . 1961

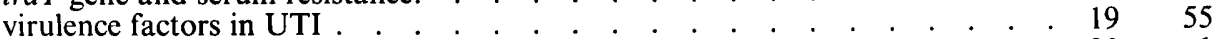

Pulmonary trichomoniasis . . . . . . . . . . . . . . . . . . . . . $20 \quad 1$

Q fever, antibody response . . . . . . . . . . . . . . . . . . . . . . . . . . . $\quad . \quad 19281$

Resistance to antibiotics

aminoglycosides, in enterococci . . . . . . . . . . . . . . . . . . . . . 20187

aminoglycosides, in S. aureus . . . . . . . . . . . . . . . . . . . . . . . . . . . . .

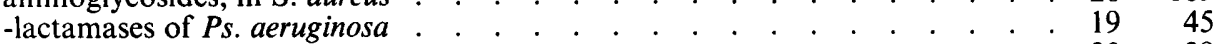

methicillin in S. aureus . . . . . . . . . . . . . . . . . . . . . . . . . . . . . .

methicillin in S. aureus . . . . . . . . . . . . . . . . . . . . . . . . . . . 2020139

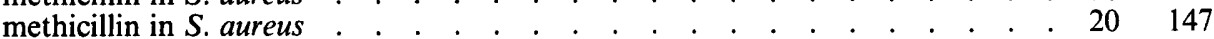

methicillin in $S$. aureus . . . . . . . . . . . . . . . . . . . . . . . . . . . . .

methicillin and gentamicin in S. aureus . . . . . . . . . . . . . . . 20157

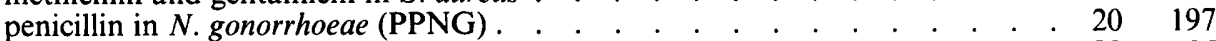

penicillin in $S$. aureus . . . . . . . . . . . . . . . . . . . . . . . . . . . . . . . . 20325

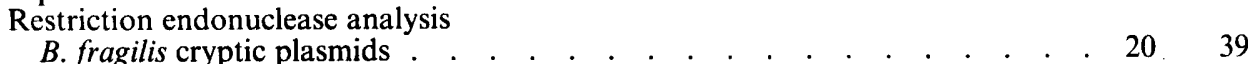

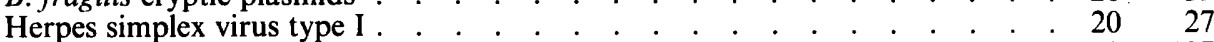

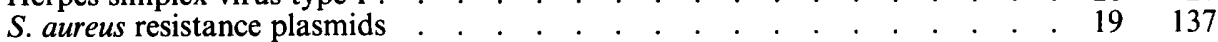

\section{Salmonella}

S. typhi, phagocytosis. . . . . . . . . . . . . . . . . . . . 19115

S. typhimurium, effect of lysolecithin on infections in mice $\quad . \quad$. . . . . . . . 19159

Serum

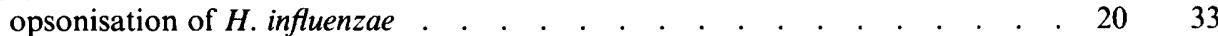

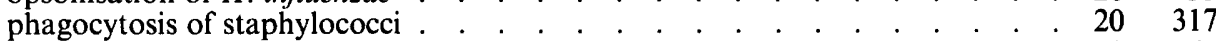

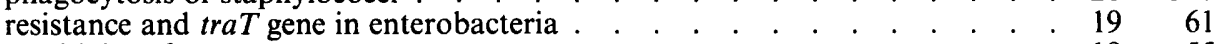

sensitivity of Proteus spp. . . . . . . . . . . . . . . . . . . . . . . . . . . . . 19

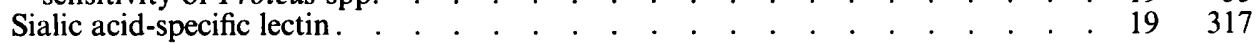

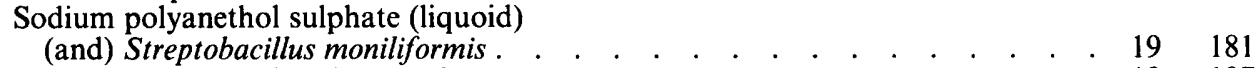

(and) streptococci and anaerobes .

Spirochaetosis in baboons . . . . . . . . . . . . . . . . . . . . . . . . . . 19359

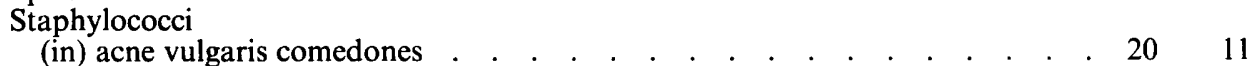

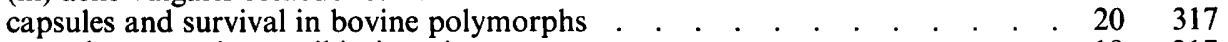

coagulase-negative, antibiotic resistance. . . . . . . . . . . . . . . . . . 19217

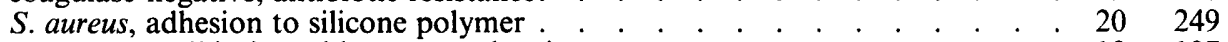

S. aureus, antibiotic and heavy metal resistance . . . . . . . . . . . . . 19137

S. aureus, antibiotic and heavy metal resistance . . . . . . . . . . . . . . 20325

S. aureus, carriage in nigerians . . . . . . . . . . . . . . . . . . . . . 19211

S. aureus, capsule expression . . . . . . . . . . . . . . . . . . . . . . . . 20275

S. aureus, (in) enteral feeds . . . . . . . . . . . . . . . . . . . . . . . . . . . . 2063

S. aureus, identification by nuclease neutralisation . . . . . . . . . . . . . . . 20392

S. aureus, lysogenicity and methicillin resistance . . . . . . . . . . . . . . . . . . 20147

S. aureus, phagocytosis . . . . . . . . . . . . . . . . . . . . . . . . 19115

S. aureus, plasmids. . . . . . . . . . . . . . . . . . . . . . . . . . . . . $\quad . \quad 20325$

$S$. aureus, plasmids in methicillin-resistant strains $\quad . \quad$. . . . . . . . . . . . . $\quad . \quad 2049$

S. aureus, resistance to nucleic acid-binding compounds . . . . . . . . . . . . 20139

S. aureus, tampon materials and toxic shock syndrome. . . . . . . . . . . . . . $\quad . \quad 2087$

Streptobacillus moniliformis, isolation from blood cultures . . . . . . . . . . . . . . . . 19181

Streptococci

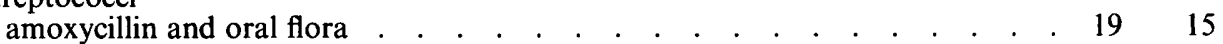

group B, lectins and phagocytosis .

inhibition of mycobacteria by viridans streptococci . . . . . . . . . . . . . $\quad$. 19227

isolation from blood cultures . . . . . . . . . . . . . . . . . . . . . . . . $\quad$. 19187

phagocytosis of Str. pyogenes and Str. pneumoniae . . . . . . . . . . . . . . 19115

streptolysin $\mathrm{O}, \mathrm{t}-$ and $\mathrm{f}$-site specificity 
transferable aminoglycoside resistance in enterococci $\begin{array}{rr}\text { PAGE } \\ 20 & 187\end{array}$

Stuart's transport medium (for anaerobes) . . . . . . . . . . . . . . . . . . . 20.373

Surface structure of Ps. aeruginosa . . . . . . . . . . . . . . . . . . . . . . . . . . . $\quad . \quad 19375$

Synergy, Esch. coli and B. fragilis $\quad . \quad$. . . . . . . . . . . . . . . . . . . . . . . . . 19325

Tampon materials, effect on S. aureus . . . . . . . . . . . . . . . . . . 2087

Tetracycline

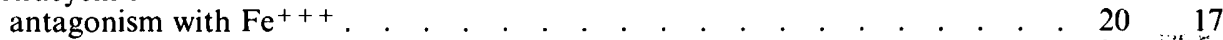

experimental candidosis in rats. . . . . . . . . . . . . . . . . . . 20 291

Toxic shock syndrome, tampon materials and S. aureus . . . . . . . . . . . . . . $20 \quad 87$

Toxins (see also enterotoxins)

(of) Bord. pertussis. . . . . . . . . . . . . . . . . . . . . . . . . . . . . . . 1939

Transduction of aminoglycoside resistance in S. aureus . . . . . . . . . . . . 20169

Tuberculosis

biodegradable implants for rifampicin . . . . . . . . . . . . . . . . . . . . . 20387

detection of antigens and antibodies in CSF . . . . . . . . . . . . . . . . . 20239

Urinary tract infection

Esch. coli adhesion to uro-epithelial cells . . . . . . . . . . . . . . . 20335

UV radiation

effect on rotavirus morphology

Varicella-zoster virus, immunity in adults . . . . . . . . . . . . . . . . . 20105

Virulence factors

Bord. bronchiseptica in piglets . . . . . . . . . . . . . . . . . . . 19247

(of) Proteus spp. . . . . . . . . . . . . . . . . . . . . . . . . . . . . . . . . . . . . . . . 1953

Y. enterocolitica invasiveness test . . . . . . . . . . . . . . . . . . . . 19237

$Y$. pestis and Y.pseudotuberculosis, plasmids, protection and virulence . . . 20225

Virus transmission by jet injection . . . . . . . . . . . . . . . . . . . 20393

Yeasts (see also Candida)

identification by disk-diffusion tests . . . . . . . . . . . . . . . . . . . . . . . 20307

Yersiniae

Y. enterocolitica, in gnotobiotic piglets . . . . . . . . . . . . . . . . . . . . . . 19297

$Y$. enterocolitica, hydrophobicity and epithelial cells. . . . . . . . . . . . . . 19309

$Y$. enterocolitica, invasiveness (mouse test) . . . . . . . . . . . . . . . . . . . . 19237

$Y$. pestis and $Y$. pseudotuberculosis, plasmids, protection and virulence . . . 20225 